\title{
Combining dark matter detectors and electron-capture sources to hunt for new physics in the neutrino sector
}

\author{
Pilar Coloma, Patrick Huber and Jonathan M. Link \\ Center for Neutrino Physics, Physics Department, Virginia Tech, \\ 850 West Campus Dr, Blacksburg, VA 24061, U.S.A. \\ E-mail: pcoloma@vt.edu, pahuber@vt.edu, jmlink@vt.edu
}

\begin{abstract}
In this letter we point out the possibility to study new physics in the neutrino sector using dark matter detectors based on liquid xenon. These are characterized by very good spatial resolution and extremely low thresholds for electron recoil energies. When combined with a radioactive $\nu_{e}$ source, both features in combination allow for a very competitive sensitivity to neutrino magnetic moments and sterile neutrino oscillations. We find that, for realistic values of detector size and source strength, the bound on the neutrino magnetic moment can be improved by an order of magnitude with respect to the present value. Regarding sterile neutrino searches, we find that most of the gallium anomaly could be explored at the $95 \%$ confidence level just using shape information.
\end{abstract}

Keywords: Beyond Standard Model, Neutrino Physics

ARXIV EPRINT: 1406.4914 


\section{Contents}

1 Introduction 1

2 Constraints on the neutrino magnetic moment 2

3 Sterile neutrino searches 4

$\begin{array}{lll}4 & \text { Results for larger exposure } & 6\end{array}$

$\begin{array}{llr}5 & \text { Summary and conclusions } & 6\end{array}$

\section{Introduction}

Neutrinos have long been a rich hunting ground for physics beyond the Standard Model (BSM). In fact, neutrino mass is so far the only BSM physics that has been established in laboratory experiments. Astrophysical evidence of dark matter suggests the existence of BSM particles, which have nevertheless not been observed yet. Among all feasible candidates, weakly interacting massive particles (WIMPs) are theoretically rather appealing. These may be observable through their interactions within detectors, as the earth moves through the sea of WIMPs. This possibility has triggered a cornucopia of experimental efforts of direct dark matter detection [1].

In this letter we examine the physics potential of combining a liquid xenon (LXe) detector, designed to search for WIMP dark matter, with an intense electron-capture neutrinos source in order to look for neutrino magnetic moments $(\nu \mathrm{MM})$ and other new physics in $\nu_{e} e^{-}$elastic scattering. The idea of looking for new physics in the neutrino sector using dark matter detectors has been proposed before in the literature, see for instance refs. [25]. Direct dark matter detection relies on observing nuclear recoils with electron-equivalent energy down to $\sim 1 \mathrm{keV}$. Due to the small values expected for the dark matter interaction cross section, large detector masses and low background levels are also required. A LXe time projection chamber (TPC) can provide a large volume, low detection thresholds (sub-keV) and a very low background rate at the energies of interest. At the same time the electron density is higher in xenon than in any other stable noble gas, thus providing the largest possible target density in any given volume near the source. The idea of using liquid noble gas detectors to search for $\nu \mathrm{MM}$ was first suggested by Vogel and Engel [6], but never developed. As a by-product we also find non-negligible sensitivity to sterile neutrino oscillations in the $\Delta m^{2} \sim 1 \mathrm{eV}^{2}$ range suggested by recent terrestrial experiments [7].

When a nucleus decays via electron-capture almost all of the available energy goes into a mono-energetic neutrino. Among possible nuclei which decay via electron-capture, ${ }^{51} \mathrm{Cr}$ offers several practical advantages: it is readily produced by thermal neutron capture [8], 
has a mean lifetime of 39.96 days and produces two mono-energetic neutrino lines at $750 \mathrm{keV}$ $(90 \%)$ and $430 \mathrm{keV}(10 \%)$. Mega-curie-scale ${ }^{51} \mathrm{Cr}$ sources have been produced in the past and used to calibrate the gallium radiochemical solar neutrino detectors GALLEX [9, 10] and SAGE [11].

\section{Constraints on the neutrino magnetic moment}

In the presence of a $\nu \mathrm{MM}$, the differential cross section for $\nu_{e} e^{-}$elastic scattering can be written as

$$
\left(\frac{d \sigma}{d T}\right)_{\mathrm{tot}}=\left(\frac{d \sigma}{d T}\right)_{\mathrm{SM}}+\frac{\pi \alpha^{2}}{m_{e}^{2}}\left(\frac{1}{T}-\frac{1}{E_{\nu}}\right)\left(\frac{\mu_{\nu}}{\mu_{B}}\right)^{2},
$$

where $m_{e}$ is the electron mass, $T$ is the electron recoil energy, $E_{\nu}$ is the neutrino energy and $\mu_{\nu}$ is the $\nu \mathrm{MM}$ in Bohr magnetons $\left(\mu_{B}\right)$. The term proportional to $\mu_{\nu}$ produces an increase in the number of events at low electron recoil energies. This makes two-phase LXe TPCs [12-17], with their low-energy detection threshold, ideal detectors for such a search. Currently, the lowest bounds on $\nu \mathrm{MM}$ come from astrophysical observations [18]: $\mu_{\nu} \lesssim 3 \times 10^{-12} \mu_{B}$. The best constraint from terrestrial experiments, on the other hand, has been obtained by the GEMMA experiment, $\mu_{\nu}<2.9 \times 10^{-11} \mu_{B}$ at $90 \%$ CL [19]. In the SM, $\nu$ MMs are expected to be many orders of magnitude below present bounds, yet many extensions of the SM produce an enhancement of the $\nu \mathrm{MM}$, see for instance ref. [20] and references therein.

For our sensitivity estimate, we assume a data taking period of 100 days, using a ${ }^{51} \mathrm{Cr}$ source with initial strength of $5 \mathrm{MCi}$. Our choice for the strength of the source is based on simulations conducted for the SOX experiment [21] of the GALLEX enriched ${ }^{50} \mathrm{Cr}$ material [8] irradiated in the High Flux Isotope Reactor at Oak Ridge National Laboratory. We consider a generic LXe detector, but for definiteness we chose a design similar to the proposed LZ detector [17, 22]. We assume a cylindrical fiducial volume with equal diameter and height, $h=1.38 \mathrm{~m}$, which contains $\sim 6$ tons of LXe, and we assume that the radioactive source is placed $1 \mathrm{~m}$ below the fiducial volume, along the central axis of the cylinder. Neutrinos are detected via electron elastic scattering in the detector, see eq. (2.1). Under these assumptions, a total of 12,518 signal events are expected for a 100 day run. Regarding backgrounds, we have considered contributions from solar neutrino interactions, ${ }^{222} \mathrm{Rn}$ and ${ }^{85} \mathrm{Kr}$ decay, and the ${ }^{136} \mathrm{Xe} 2 \nu \beta \beta$ decay. Following ref. [23], the solar neutrino background is estimated to be 1.05 counts per ton and day for $p p$ neutrinos and 0.51 counts per ton and day for ${ }^{7} \mathrm{Be}$ neutrinos. The ${ }^{85} \mathrm{Kr}$ and ${ }^{136} \mathrm{Xe}$ backgrounds have been taken directly from figure 2 in ref. [23] and rescaled according to our run length and detector mass, while for ${ }^{222} \mathrm{Rn}$ we assume that a goal of $0.1 \mu \mathrm{Bq} / \mathrm{kg}$ can be achieved [23], about a factor of 30 reduction with respect to what has been achieved for EXO-200 [24].

Finally, an important source of background could come from the source itself. In $10 \%$ of ${ }^{51} \mathrm{Cr}$ decays there is a $320 \mathrm{keV}$ gamma, which can easily be shielded with just a few $\mathrm{cm}$ of tungsten. However, impurities present in the chromium prior to irradiation, can lead to the production of $\mathrm{MeV}$ gamma emitters [11, 25]. These will require significant additional shielding to be reduced down to an acceptable level. We base our calculation of 

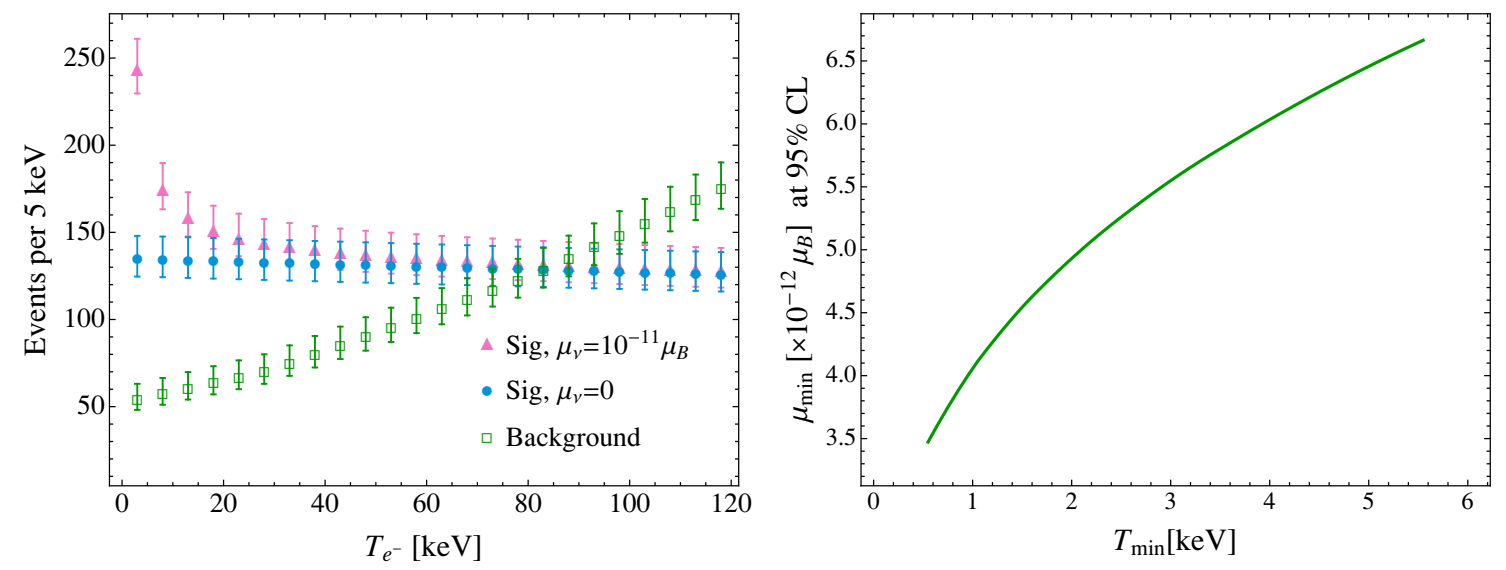

Figure 1. Left panel: number of events (per $5 \mathrm{keV}$ ) as a function of the recoil energy of the electron. Results for the signal are shown with (pink triangles) and without (blue circles) a $\nu$ MM. The expected background event rates are also shown for comparison (green squares). Right panel: the achievable bound on the $\nu \mathrm{MM}$ (at the $95 \% \mathrm{CL}$ ) as a function of the low-energy threshold on electron recoil energy.

this background on the measured gamma activity of the GALLEX source [25]. Our source is assumed to be shielded by a $17 \mathrm{~cm}$ thick tungsten layer, which, when combined with $70 \mathrm{~cm}$ of LXe (present between the tungsten shield and the edge of the detector), provides an attenuation of $10^{-11}$ for a $1.5 \mathrm{MeV}$ gamma. Nevertheless, with $10^{13}$ gammas emitted per day (in all directions), we expect about 10 to pass through the shielding and Compton scatter in the detector fiducial volume. Most of these would deposit energy in excess of the maximum from a ${ }^{51} \mathrm{Cr}$ neutrino, though. Further suppression could come from a veto on mulit-site Compton scattering events. We estimate the surviving background in the fiducial volume from source gammas to be less than 1 event per day at the start of the data taking, and, since $\sim 95 \%$ of $\mathrm{MeV}$ gammas come from short-lived isotopes (such as ${ }^{64} \mathrm{Cu}$, ${ }^{77} \mathrm{Ge}$ and ${ }^{24} \mathrm{Na}$ ), this rate should rapidly decay with time. Therefore, we will neglect these events in our analysis, but we note that care must be taken in the preparation of the ${ }^{50} \mathrm{Cr}$ source material to ensure the required level of purity is reached.

To constrain the $\nu \mathrm{MM}$, the analysis is done in terms of the recoil energy of the electron only. The recoil energies are smeared on an event-by-event basis according to a Gaussian with $\sigma(T)=0.20 \sqrt{T}$. Generally, we find that the energy resolution does not have a significant impact on the $\nu \mathrm{MM}$ sensitivity. A Poissonian $\chi^{2}$ is constructed using $0.1 \mathrm{keV}$ wide bins in $T$, from $2 \mathrm{keV}$ to $140 \mathrm{keV}$ unless otherwise stated. In this energy range a total of 3,656 signal events are expected, together with a total of 3,450 background events. The distribution of signal and background events in electron recoil energy is shown in figure 1 (left). Reducing the backgrounds does not significantly improve the sensitivity to this observable since in the low-energy region, where the $\nu \mathrm{MM}$ enhancement is expected, the background rate is already quite low. On the other hand, we find the low-energy threshold to be the most relevant parameter in this analysis (as expected from eq. (2.1)). Figure 1 (right) shows the dependence of the sensitivity as a function of detector threshold. With 
a fairly conservative threshold of $2 \mathrm{keV}$ the $95 \%$ CL bound is $\mu_{\nu}<4.9 \times 10^{-12} \mu_{B}$. Such values would yield an improvement of a factor of 5 over the currently best terrestrial limit.

\section{Sterile neutrino searches}

A second possible application of the setup studied here would be the search for light sterile neutrinos. Sterile neutrinos arise in most models of neutrino mass generation. Searches for oscillations between active and sterile neutrinos have been conducted using many combinations of neutrino sources, detectors and oscillation channels [7]. Experimental results are inconclusive, with strong tension between different data sets (see, e.g., refs. [7, 26, 27]). In particular, an analysis of GALLEX and SAGE data shows an apparent deficit of events which is consistent with oscillations involving a sterile neutrino with a squared mass difference with the active states $\Delta m^{2} \sim 1 \mathrm{eV}^{2}$ and a mixing angle such that $\sin ^{2} 2 \theta \sim 0.1$ [28], which is commonly referred to as the gallium anomaly. The smoking gun of a sterile neutrino would be to observe events following an oscillating pattern in $L / E_{\nu}$, the distance traveled by the neutrino divided by its energy. A monochromatic neutrino source reduces the oscillating pattern to a pure function of $L$. Given the energy of the primary ${ }^{51} \mathrm{Cr}$ neutrino the oscillation length at $\Delta m^{2}=1 \mathrm{eV}^{2}$ would be $\sim 90 \mathrm{~cm}$ and thus the oscillating pattern would be observable inside a meter-scale detector. The expected spatial resolution in LXe TPCs is at the sub-cm level, which makes them ideal candidates for such an experiment.

In our analysis, we have adopted a phenomenological approach based on a $3+1$ framework, where there is only one extra sterile state at the eV scale. In this framework, the $\nu_{e}$ disappearance oscillation probability can be expressed in terms of one mixing angle and one mass squared splitting only, as:

$$
P_{e e}=1-\sin ^{2} 2 \theta_{e e} \sin ^{2}\left(\frac{\Delta m_{41}^{2} L}{4 E_{\nu}}\right),
$$

where $E_{\nu}$ is the neutrino energy, $L$ is the distance between the source and the interaction point in the detector, $\Delta m_{41}^{2} \equiv m_{4}^{2}-m_{1}^{2}$ and $\theta_{e e}$ is an effective mixing angle. The sensitivity to sterile neutrino oscillations is computed using the distance between the source and the interaction point $(L)$ as the main variable in the analysis. A binned Gaussian $\chi^{2}$ is built using $3 \mathrm{~cm}$ wide bins. The detector is assumed to have a constant spatial resolution of $1 \mathrm{~cm}$; however, the largest uncertainty in $L$ comes from the shape and size of the radioactive source itself. In the present work, we assume that the source will be a cylinder with both height and diameter equal to $14 \mathrm{~cm}$. To achieve such a compact source will require that the chromium material be enriched to $\sim 95 \%$ in ${ }^{50} \mathrm{Cr}$. This is much higher than what was achieved by GALLEX [8], but similar to what was reached by SAGE [11]. A smearing function was generated, by a Monte Carlo calculation, that simultaneously accounts for the finite source size and the detector resolution. Two nuisance parameters are added to the $\chi^{2}$ for signal and background normalization uncertainties. A Gaussian penalty term (or pull-term) is added to the $\chi^{2}$ for the background uncertainty and marginalization is performed over both nuisance parameters. Unless otherwise stated, no constraint is 

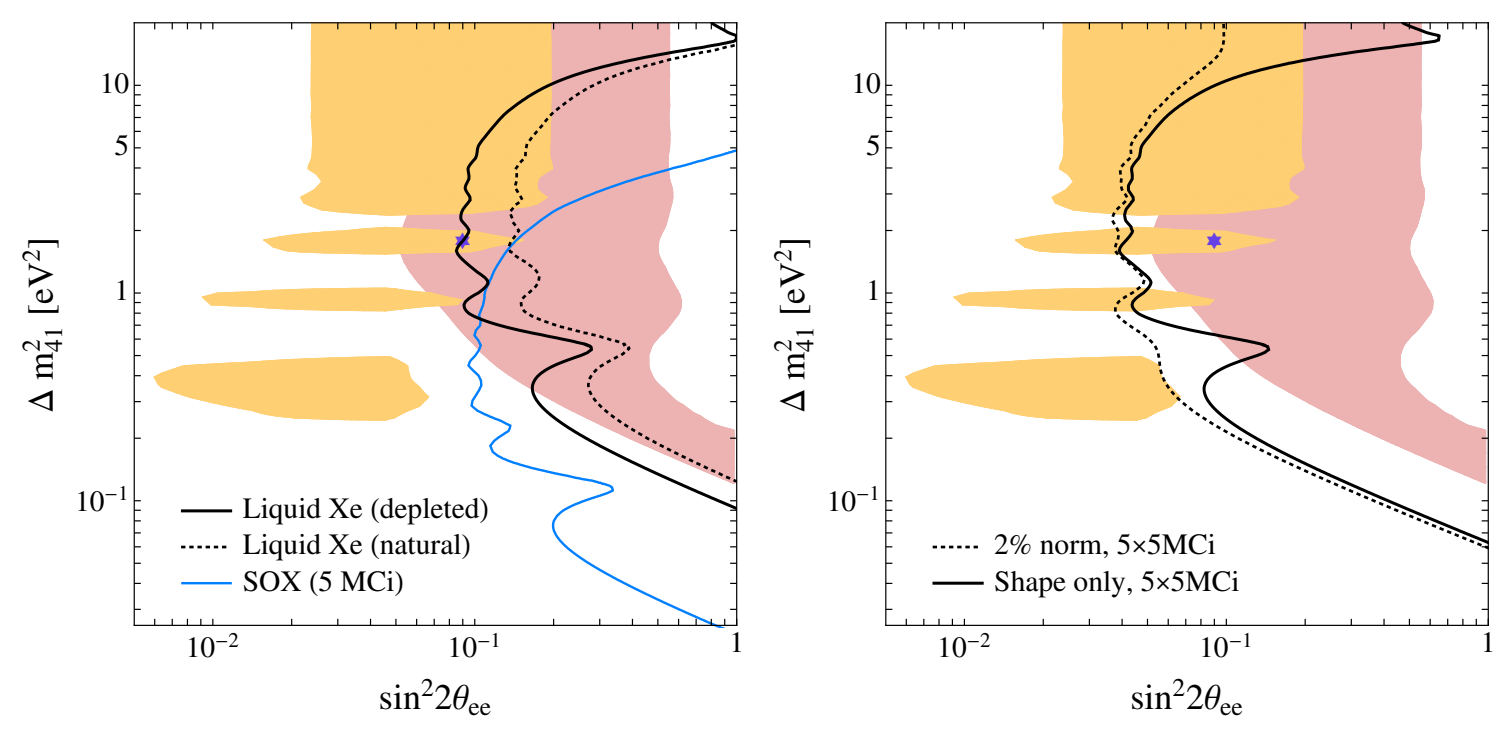

Figure 2. Sensitivity to sterile neutrino oscillations as a function of $\sin ^{2} 2 \theta_{e e}$ and $\Delta m_{41}^{2}$. The parameter space to the right of each line would be excluded at 95\% CL (2 d.o.f.). The shaded areas show the $95 \%$ CL allowed regions for the reactor (yellow) and gallium (pink) anomalies from a global fit to the $3+1$ scenario, while the star indicates the best fit point from a combined fit to both anomalies [26]. Left panel: expected sensitivity using shape information only (i.e., normalization is left completely free). Black lines show the expected contours for the LXe experiment described in the text. For comparison, the solid blue line shows the SOX sensitivity using shape information alone. In both cases a $5 \mathrm{MCi}$ radioactive ${ }^{51} \mathrm{Cr}$ source is assumed. Right panel: expected sensitivity for the LXe experiment with five source deployments. Results in this panel are shown from analyses using shape information only (solid lines) and shape plus normalization (dotted lines).

assumed for the flux normalization and it is therefore left completely free during the fit. For the backgrounds, an uncertainty of $0.5 \%$ is assumed. We expect this could be achieved by using the data collected during the source-free operation of the detector corresponding to the the dark matter search. The process is repeated for each point in the $\left(\sin ^{2} 2 \theta, \Delta m^{2}\right)$ parameter space. Since the low-end threshold for the electron recoil energy is not expected to have a great impact on the sterile neutrino analysis, it is set to $5 \mathrm{keV}$ in this case.

The results for the sterile neutrino sensitivity are shown in figure 2. Since the sensitivity mainly comes from a shape analysis, this measurement is far from being limited by systematics. We find the main limiting factor to be the ${ }^{136} \mathrm{Xe}$ background. The expected number of background events (across the full energy range) is around 51,130, from which around 44,000 are from ${ }^{136} \mathrm{Xe} 2 \nu \beta \beta$ decays. This background can be reduced by using LXe depleted in ${ }^{136} \mathrm{Xe}$, though. The sensitivities for both possibilities are shown in figure 2 (left). For contrast, we also compare the LXe sensitivity to that of Borexino/SOX [21] using a comparable, $5 \mathrm{MCi}{ }^{51} \mathrm{Cr}$ source (the SOX proposal is based on $10 \mathrm{MCi}$ ). The complementarity in $\Delta m^{2}$ coverage of the two experiments is evident. In the case of LXe, the relatively high spatial resolution improves sensitivity at high $\Delta m^{2}$, while for SOX, the relatively large detector volume, or range in $L$, improves the reach at low $\Delta m^{2}$. It is remarkable that, for a depleted Xe experiment, most of the region favored by the gallium 
anomaly is covered with shape information alone. Therefore, if the gallium anomaly is correct this configuration would, with high likelihood, confirm it by a clear observation of the oscillatory pattern.

\section{Results for larger exposure}

Both the sterile neutrino and $\nu \mathrm{MM}$ searches are statistics limited and would be improved by repeated redeployments of the ${ }^{51} \mathrm{Cr}$ source. Repeated deployment has a precedent in GALLEX, which irradiated and deployed the same source material twice [10]. We will consider five deployments, each identical in source strength and duration to our previously considered single deployment.

In the case of the $\nu \mathrm{MM}$ search, the sensitivity with increased statistics is remarkable. At $95 \% \mathrm{CL}$, assuming a low-energy threshold of $2 \mathrm{keV}$, the corresponding bound is pushed down to $\mu_{\nu}=3.31 \times 10^{-12} \mu_{B}$. If a lower threshold can be achieved, even the astrophysical limit would be surpassed.

The sensitivity to sterile neutrino oscillations with increased statistics is shown in figure 2 (right). In this case, we assume $90 \%$ depleted Xe, and show sensitivities from both a shape-only analysis, and an analysis with the shape information plus a $2 \%$ uncertainty in the normalization. As can be seen in the comparison of the two bounds, no major improvement is expected from imposing the normalization constraint, since the information comes from observing the oscillating pattern in the detector. Only in case of large $\Delta m_{41}^{2}$, where the oscillation is averaged out, would a normalization constraint help. According to our results, after five deployments the full gallium anomaly as well as a sizable region of the reactor anomaly would be covered at $95 \%$ CL.

Obviously, with 10,000-50,000 $\nu_{e} e^{-}$elastic scattering events with momentum transfers in the $100 \mathrm{keV}$ range a number of precision tests of the electro-weak sector of the SM become possible. Assuming an absolute normalization at the $1 \%$ level and statistical errors at or below $1 \%$, overall accuracies for the total cross section of 1-2\% appear feasible, providing constraints on the weak mixing angle and its running.

\section{Summary and conclusions}

To summarize, our results indicate that the combination of a large liquid xenon detector, designed and built to search for dark matter, with a Mega-curie scale electron capture neutrino source would provide excellent reach in the search for the neutrino magnetic moment, exceeding the current laboratory bounds by at least a factor of 5 . With repeated source deployments, such an experiment would even be competitive with the best astrophysical limits. Moreover, this combination would allow a test of the reactor and gallium anomalies; specifically, their interpretation as oscillations due to an $\mathrm{eV}$-scale sterile neutrino. Its reach would be complementary to other source proposals with sensitivity to the oscillating pattern over almost the entire interesting range of $\Delta m^{2}$. Clearly, a detailed technical feasibility study is required, but so far no major technological obstacles have been identified. 


\section{Acknowledgments}

This work has been supported by the U.S. Department of Energy under award numbers DE-SC0003915 and DE-SC0009973. We would like to thank the authors of ref. [26] for sharing with us their results for the global fit of the reactor and gallium anomalies.

Open Access. This article is distributed under the terms of the Creative Commons Attribution License (CC-BY 4.0), which permits any use, distribution and reproduction in any medium, provided the original author(s) and source are credited.

\section{References}

[1] D. Bauer et al., Dark matter in the coming decade: complementary paths to discovery and beyond, arXiv:1305.1605 [INSPIRE].

[2] M. Pospelov, Neutrino physics with dark matter experiments and the signature of new baryonic neutral currents, Phys. Rev. D 84 (2011) 085008 [arXiv:1103.3261] [InSPIRE].

[3] R. Harnik, J. Kopp and P.A.N. Machado, Exploring $\nu$ signals in dark matter detectors, JCAP 07 (2012) 026 [arXiv: 1202.6073] [INSPIRE].

[4] M. Pospelov and J. Pradler, Elastic scattering signals of solar neutrinos with enhanced baryonic currents, Phys. Rev. D 85 (2012) 113016 [arXiv:1203.0545] [INSPIRE].

[5] M. Pospelov and J. Pradler, Dark matter or neutrino recoil? Interpretation of recent experimental results, Phys. Rev. D 89 (2014) 055012 [arXiv:1311.5764] [InSPIRE].

[6] P. Vogel and J. Engel, Neutrino electromagnetic form-factors, Phys. Rev. D 39 (1989) 3378 [INSPIRE].

[7] K.N. Abazajian et al., Light sterile neutrinos: a white paper, arXiv:1204.5379 [INSPIRE].

[8] M. Cribier et al., Production of a $62 \mathrm{~PB} \mathrm{P}^{51} \mathrm{Cr}$ low-energy neutrino source for GALLEX, Nucl. Instrum. Meth. A 378 (1996) 233 [INSPIRE].

[9] GALLEX collaboration, P. Anselmann et al., First results from the ${ }^{51} \mathrm{Cr}$ neutrino source experiment with the GALLEX detector, Phys. Lett. B 342 (1995) 440 [INSPIRE].

[10] GALLEX collaboration, W. Hampel et al., Final results of the ${ }^{51} \mathrm{Cr}$ neutrino source experiments in GALLEX, Phys. Lett. B 420 (1998) 114 [INSPIRE].

[11] SAGE collaboration, J.N. Abdurashitov et al., Measurement of the response of the Russian-American gallium experiment to neutrinos from a ${ }^{51} \mathrm{Cr}$ source, Phys. Rev. C 59 (1999) 2246 [hep-ph/9803418] [INSPIRE].

[12] ZEPLIN-III collaboration, V.N. Lebedenko et al., Limits on the spin-dependent WIMP-nucleon cross-sections from the first science run of the ZEPLIN-III experiment, Phys. Rev. Lett. 103 (2009) 151302 [arXiv:0901.4348] [INSPIRE].

[13] XENON100 collaboration, E. Aprile et al., The XENON100 dark matter experiment, Astropart. Phys. 35 (2012) 573 [arXiv:1107.2155] [INSPIRE].

[14] LUX collaboration, D.S. Akerib et al., The Large Underground Xenon (LUX) experiment, Nucl. Instrum. Meth. A 704 (2013) 111 [arXiv:1211.3788] [INSPIRE].

[15] XENON1T collaboration, E. Aprile, The XENON1T dark matter search experiment, Springer Proc. Phys. C12-02-22 (2013) 93 [arXiv:1206.6288] [INSPIRE]. 
[16] DARWIN Consortium collaboration, L. Baudis, DARWIN: dark matter WIMP search with noble liquids, J. Phys. Conf. Ser. 375 (2012) 012028 [arXiv:1201.2402] [INSPIRE].

[17] D.C. Malling et al., After LUX: the LZ program, arXiv:1110.0103 [INSPIRE].

[18] G.G. Raffelt, Limits on neutrino electromagnetic properties: an update, Phys. Rept. 320 (1999) 319 [INSPIRE].

[19] A. Beda et al., The results of search for the neutrino magnetic moment in GEMMA experiment, Adv. High Energy Phys. 2012 (2012) 350150.

[20] C. Broggini, C. Giunti and A. Studenikin, Electromagnetic properties of neutrinos, Adv. High Energy Phys. 2012 (2012) 459526 [arXiv:1207.3980] [INSPIRE].

[21] Borexino collaboration, G. Bellini et al., SOX: Short distance neutrino Oscillations with BoreXino, JHEP 08 (2013) 038 [arXiv:1304.7721] [INSPIRE].

[22] LZ collaboration, private communication.

[23] L. Baudis et al., Neutrino physics with multi-ton scale liquid xenon detectors, JCAP 01 (2014) 044 [arXiv: 1309.7024] [INSPIRE].

[24] EXO-200 collaboration, J.B. Albert et al., Improved measurement of the $2 \nu \beta \beta$ half-life of ${ }^{136}$ Xe with the EXO-200 detector, Phys. Rev. C 89 (2014) 015502 [arXiv:1306.6106] [INSPIRE].

[25] E. Bellotti et al., Measurement of the gamma activity induced by neutron activation in the GALLEX neutrino source, Nucl. Instrum. Meth. B 100 (1995) 199.

[26] J. Kopp, P.A.N. Machado, M. Maltoni and T. Schwetz, Sterile neutrino oscillations: the global picture, JHEP 05 (2013) 050 [arXiv: 1303.3011] [INSPIRE].

[27] C. Giunti and M. Laveder, Status of 3+1 neutrino mixing, Phys. Rev. D 84 (2011) 093006 [arXiv: 1109.4033] [INSPIRE].

[28] M.A. Acero, C. Giunti and M. Laveder, Limits on $\nu_{e}$ and $\bar{\nu}_{e}$ disappearance from gallium and reactor experiments, Phys. Rev. D 78 (2008) 073009 [arXiv:0711.4222] [INSPIRE]. 